University of Wollongong

Research Online

Faculty of Engineering and Information

Faculty of Engineering and Information

Sciences - Papers: Part B

Sciences

2020

Pricing variance and volatility swaps with stochastic volatility, stochastic interest rate and regime switching

Sha Lin

Zhejiang Gongshang University, s1945@uowmail.edu.au

Xinjiang He

University of Wollongong, xinjiang@uow.edu.au

Follow this and additional works at: https://ro.uow.edu.au/eispapers1

Part of the Engineering Commons, and the Science and Technology Studies Commons

Research Online is the open access institutional repository for the University of Wollongong. For further information contact the UOW Library: research-pubs@uow.edu.au 


\title{
Pricing variance and volatility swaps with stochastic volatility, stochastic interest rate and regime switching
}

\author{
Abstract \\ In this paper, we propose a two-factor Heston-CIR hybrid model for the pricing of variance and volatility \\ swaps, by introducing the second regime switching factor into the Heston-CIR hybrid model. While this \\ model is closer to reality, taking advantages of the Heston stochastic volatility, CIR stochastic interest \\ rate and regime switching, it has a more complicated structure and thus leads to extra difficulty in finding \\ analytical solutions. Albeit difficult, we have still managed to present analytical pricing formulae for \\ variance and volatility swaps, based on the derived forward characteristic function in a series form. The \\ series solutions are accompanied by a radius of convergence to ensure its safe application, and their fast \\ convergence demonstrated through numerical experiments facilitates the implementation in practice.

\section{Disciplines \\ Engineering | Science and Technology Studies}

\section{Publication Details} \\ Lin, S. \& He, X. (2020). Pricing variance and volatility swaps with stochastic volatility, stochastic interest \\ rate and regime switching. Physica A: Statistical Mechanics and its Applications, 537 \\ 122714-1-122714-14.
}




\title{
Pricing variance and volatility swaps with stochastic volatility, stochastic interest rate and regime switching
}

\author{
Sha Lin * Xin-Jiang $\mathrm{HE}^{\dagger}$
}

\begin{abstract}
In this paper, we propose a two-factor Heston-CIR hybrid model for the pricing of variance and volatility swaps, by introducing the second regime switching factor into the Heston-CIR hybrid model. While this model is closer to reality, taking advantages of the Heston stochastic volatility, CIR stochastic interest rate and regime switching, it has a more complicated structure and thus leads to extra difficulty in finding analytical solutions. Albeit difficult, we have still managed to present analytical pricing formulae for variance and volatility swaps, based on the derived forward characteristic function in a series form. The series solutions are accompanied by a radius of convergence to ensure its safe application, and their fast convergence demonstrated through numerical experiments facilitates the implementation in practice.
\end{abstract}

\section{AMS(MOS) subject classification.}

Keywords. Two-factor Heston-CIR hybrid model, variance and volatility swaps, regime switching, analytical, convergence.

\footnotetext{
${ }^{*}$ School of Finance, Zhejiang Gongshang University, Hangzhou 310018, China.

${ }^{\dagger}$ Corresponding author. School of Mathematics and Applied Statistics, University of Wollongong NSW 2522, Australia. Email: xinjiang@uow.edu.au.
} 


\section{Introduction}

Variance and volatility swaps are two of the most popular volatility derivatives that are able to help investors effectively manage financial risk as they do not have to invest the assets themselves. Due to their popularity, the accurate and efficient determination of their prices is really demanding, and has received a lot of attention. For example, the pricing of variance and volatility swaps have been considered under various models $[9,15,17]$, while Carr \& Lee [5, 6] went even further by presenting model independent results, by assuming that the realized variance or volatility is continuously sampled. However, the assumption of continuous sampling is apparently not appropriate as the realized variance or volatility is discretely sampled in real markets, and the obtained results can only be treated as approximations to the real prices, while there is no guarantee of the quality of these approximations.

In this sense, using discrete sampling in pricing the two swap contracts is much more favored as it is closer to practice. However, it should be noted that no model independent results are no longer available when evaluating discretely-sampled variance and volatility swaps, and the choice of an appropriate model for the underlying price is also vital. As one may be aware that although the celebrated Black-Scholes model [3] is widely adopted in derivative pricing, it contains a few severe model flaws, such as the assumption of constant volatility and constant interest rate. This prompts the researchers to develop various models and work on the derivative pricing problems under these models. A natural modification to the Black-Scholes model is to add non-constant volatility and there have already been a few results on the evaluation of discretely-sampled variance and volatility swaps under models included in this category. In specific, Little \& Pant [19] adopted the finite difference method in pricing discretely sampled variance swaps when the underlying price follows the local volatility model through a dimension-reduction approach, while Zhu \& Lian $[21,22]$ provided analytical pricing formulae for variance and volatility swaps when the volatility of the underlying price is assumed to be stochastic, following the well-known 
Heston model [16].

Despite these appealing results, it has been pointed out by a few authors that stochastic volatility models may not be adequate in describing the real market behavior, and researchers are still trying to establish more sophisticated models, the attempts of which include local regime-switching models $[12,14]$, time-dependent stochastic volatility models [8] and regime-switching stochastic volatility models [11]. Of course, making the interest rate in stochastic volatility models another random variable is also very popular as the model performance is demonstrated to be improved when incorporating stochastic interest rate [1], and various hybrid models have been formulated, such as the Heston-CIR hybrid model [13], a combination of the Heston volatility model and CIR (Cox-Ingersoll-Ross) interest rate model, and the Stein-Stein-Hull-White hybrid model [20], with both volatility and interest rate following the Ornstein-Uhlenbeck process. Some of these models have already been introduced into variance and volatility swaps. A typical examples is [7], where the prices of the two swap contracts are analytically determined under a regime switching Heston model.

Motivated by the existence of regime switching in real markets [10] and better results obtained after the incorporation of stochastic interest rate [18], in this paper, we propose a two-factor Heston-CIR hybrid model. This model takes the advantages of the HestonCIR hybrid model in the sense that both volatility and interest rate are stochastic following different CIR processes, which satisfy a few important properties possessed by the volatility and interest rate, such as non-negative property and mean reverting property, while at the same time accounts for the effect of regime switching on variance and volatility swap prices. While combining stochastic volatility, stochastic interest rate and regime switching together satisfies the practical demand, the complicatedness of the constructed model poses an obstacle in finding analytical solutions. Albeit difficult, we have still managed to present analytical pricing formulae for variance and volatility swaps, written in a series form. The formulae are theoretically appealing as they are equipped with a radius of convergence, 
and the formulae are also advantageous from the practical point of view as they are very quick to implement, as demonstrated through numerical experiments.

The rest of the paper is organized as follows. In Section 2, we introduce the newly proposed two-factor Heston-CIR hybrid model. In Section 3, we present the derivation details of variance and volatility swap pricing formulae. Numerical examples and discussions are presented in Section 4, followed by some concluding remarks given in the last section.

\section{The two-factor Heston-CIR hybrid model}

In this section, we propose a two-factor Heston-CIR hybrid model based on the classical Heston-CIR hybrid model, which is a combination of the Heston stochastic volatility model and CIR interest rate model, and the introduced second factor is used to enable the model to capture the effect of regime switching. In the following, the dynamics of the HestonCIR hybrid model will be firstly specified, with which one can easily see how our model is constructed.

If the underlying price, volatility and the interest rate are denoted by $S_{t}, v_{t}$ and $r_{t}$, respectively, the Heston-CIR hybrid model under a risk-neutral measure $\mathbb{Q}$ has the following expression

$$
\begin{aligned}
\frac{d S_{t}}{S_{t}} & =r_{t} d t+\sqrt{v_{t}} d W_{1, t}^{S}, \\
d v_{t} & =k\left(\theta-v_{t}\right) d t+\sigma \sqrt{v_{t}} d W_{t}^{v} \\
d r_{t} & =\alpha\left(\beta-r_{t}\right) d t+\eta \sqrt{r_{t}} d W_{t}^{r}
\end{aligned}
$$

where $W_{1, t}^{S}$ and $W_{t}^{v}$ are two standard Brownian motions with correlation $\rho$, while they are independent of another Brownian motion $W_{t}^{r}$. As mentioned earlier, in order to take advantage of multi-factor stochastic volatility models and the effect of regime switching, we introduce a regime switching factor into the Heston-CIR hybrid model so that our model 
dynamics are

$$
\begin{aligned}
\frac{d S_{t}}{S_{t}} & =r_{t} d t+\sqrt{v_{t}} d W_{1, t}^{S}+\xi_{X_{t}} d W_{2, t}^{S} \\
d v_{t} & =k\left(\theta-v_{t}\right) d t+\sigma \sqrt{v_{t}} d W_{t}^{v} \\
d r_{t} & =\alpha\left(\beta-r_{t}\right) d t+\eta \sqrt{r_{t}} d W_{t}^{r}
\end{aligned}
$$

with $W_{2, t}^{S}$ being another standard Brownian motion being independent of the other three. $\xi_{X_{t}}$ is a regime switching parameter, controlled by a Markov chain $X_{t}$, whose definition ${ }^{1}$ is given by

$$
X_{t}= \begin{cases}(1,0)^{T}, & \text { when the economy is believed to be in State 1 } \\ (0,1)^{T}, & \text { when the economy is believed to be in State 2 }\end{cases}
$$

with the transition between the two states following a Poisson process

$$
P\left(t_{i j}>t\right)=e^{-\lambda_{i j} t}, i, j=1,2, i \neq j
$$

Here, $\lambda_{i j}$ represents the transition rate from State $i$ to $j$, while $t_{i j}$ stands for the time spent in State $i$ before transferring to State $j$. Under this setting, $\xi_{X_{t}}$ can be expressed as $\xi_{X_{t}}=<\bar{\xi}, X_{t}>$ if the values of the second factor in both states are put in the vector $\bar{\xi}=\left(\xi_{1}, \xi_{2}\right)^{T}$ and $<\cdot, \cdot>$ denotes the inner product of two vectors.

With the dynamics of the new model being presented in (2.2), a natural question is how variance and volatility swaps can be analytically evaluated. However, one should notice that the introduction of the regime switching factor has caused extra difficulty in finding analytical solution. In the next section, the detailed derivation for the analytical pricing formulae of variance and volatility swaps will be provided.

\footnotetext{
${ }^{1}$ For illustration purposes, we will focus on the two state Markov chain, but the extension to arbitrary but finite states can be quite straightforward.
} 


\section{Valuation of variance and volatility swaps}

In this section, the pricing of variance and volatility swaps will be discussed in details, with analytical pricing formulae presented based on the derived forward characteristic function. Before we start, it needs to be pointed out that the "price" for a variance or volatility swap contract that needs to be determined is not the value of the contract itself, but instead, it refers to the delivery price specified in the contracts, as variance and volatility swaps are nothing but forward contracts. These contracts work through the agreement reached by both parties that the long positions pay the delivery price price at expiry while they receive floating amount of the realized variance or volatility several times within the time period of the contract.

\subsection{The general pricing approach}

We begin by specifying the formulae for the values of swap contracts, $V_{v a r}$ and $V_{v o l}$, as

$V_{v a r}=E^{Q}\left[e^{-\int_{0}^{T} r_{t} d t}\left(R V_{v a r}-K_{v a r}\right) L \mid S_{0}, v_{0}, r_{0}, X_{0}\right], \quad V_{v o l}=E^{Q}\left[e^{-\int_{0}^{T} r_{t} d t}\left(R V_{v o l}-K_{v o l}\right) L \mid S_{0}, v_{0}, r_{0}, X_{0}\right]$

with $K_{v a r}$ and $K_{v o l}$ representing the delivery prices of a variance and volatility swap contract, respectively, and $L$ denoting the notional amount given in the contracts. $R V_{v a r}$ and $R V_{v o l}$ are the annualized realized variance and volatility, respectively. Due to the nature of the contacts, their values should be zero when they are initiated, leading to

$$
\begin{aligned}
& K_{v a r} E^{Q}\left[e^{-\int_{0}^{T} r_{t} d t} \mid r_{0}\right]=E^{Q}\left[e^{-\int_{0}^{T} r_{t} d t} R V_{v a r} \mid S_{0}, v_{0}, r_{0}, X_{0}\right]=E^{Q}\left[e^{-\int_{0}^{T} r_{t} d t} \mid r_{0}\right] E^{Q^{T}}\left[R V_{v a r} \mid S_{0}, v_{0}, r_{0}, X_{0}\right] \\
& K_{v o l} E^{Q}\left[e^{-\int_{0}^{T} r_{t} d t} \mid r_{0}\right]=E^{Q}\left[e^{-\int_{0}^{T} r_{t} d t} R V_{v o l} \mid S_{0}, v_{0}, r_{0}, X_{0}\right]=E^{Q}\left[e^{-\int_{0}^{T} r_{t} d t} \mid r_{0}\right] E^{Q^{T}}\left[R V_{v o l} \mid S_{0}, v_{0}, r_{0}, X_{0}\right]
\end{aligned}
$$


after making the measure transformation, with $\mathbb{Q}^{T}$ as the $T$-forward measure. Further simplification will certainly yield

$$
\begin{aligned}
K_{v a r} & =E^{Q^{T}}\left[R V_{v a r} \mid S_{0}, v_{0}, r_{0}, X_{0}\right] \\
K_{v o l} & =E^{Q^{T}}\left[R V_{v o l} \mid S_{0}, v_{0}, r_{0}, X_{0}\right]
\end{aligned}
$$

from which one can easily deduce that the delivery prices to be determined are equal to the expectations on the right hand side depending on the definitions of the realized variance and volatility. The popular choices in the literature $[7,17,21]$ are

$$
\begin{aligned}
R V_{\text {var }} & =\frac{100^{2}}{T} \sum_{i=1}^{N}\left(\frac{S_{t_{i}}-S_{t_{i-1}}}{S_{t_{i-1}}}\right)^{2} \\
R V_{\text {vol }} & =100 \sqrt{\frac{\pi}{2 N T}} \sum_{i=1}^{N}\left|\frac{S_{t_{i}}-S_{t_{i-1}}}{S_{t_{i-1}}}\right|
\end{aligned}
$$

where the number of payments for the floating amount of the realized variance or volatility is $N$, and the time interval between two subsequent payments is assumed to be equal to each other. With the substitution of the expressions of $R V_{v a r}$ and $R V_{v o l}$, it is not difficult to find that the delivery prices of variance and volatility swaps are

$$
\begin{aligned}
K_{v a r} & =E^{Q^{T}}\left[R V_{v a r}\right]=\frac{100^{2}}{T} \sum_{i=1}^{N} E^{Q^{T}}\left[\left(\frac{S_{t_{i}}-S_{t_{i-1}}}{S_{t_{i-1}}}\right)^{2} \mid S_{0}, v_{0}, r_{0}, X_{0}\right], \\
K_{v o l} & =E^{Q^{T}}\left[R V_{v o l}\right]=100 \sqrt{\frac{\pi}{2 N T}} \sum_{i=1}^{N} E^{Q^{T}}\left[\left|\frac{S_{t_{i}}-S_{t_{i-1}}}{S_{t_{i-1}}}\right| \mid S_{0}, v_{0}, r_{0}, X_{0}\right] .
\end{aligned}
$$

If we make $Z_{t}=\ln \left(S_{t}\right)$ and denote $y_{t, T}=Z_{T}-Z_{t}$, we can certainly obtain

$$
\frac{S_{t_{i}}-S_{t_{i-1}}}{S_{t_{i-1}}}=e^{y_{t_{i-1}, t_{i}}}-1,
$$


which which the delivery price of a variance swap can be calculated from

$$
K_{\text {var }}=\frac{100^{2}}{T} \sum_{i=1}^{N} E^{Q^{T}}\left[e^{2 y_{t_{i-1}, t_{i}}}-2 e^{y_{t_{i-1}, t_{i}}}+1 \mid S_{0}, v_{0}, r_{0}, X_{0}\right]
$$

while the delivery price of a volatility swap can be determined through

$$
\begin{aligned}
K_{v o l} & =100 \sqrt{\frac{\pi}{2 N T}}\left[-\int_{0}^{+\infty} p\left(y_{t_{i-1}, t_{i}}\right) d y_{t_{i-1}, t_{i}}+\int_{-\infty}^{0} p\left(y_{t_{i-1}, t_{i}}\right) d y_{t_{i-1}, t_{i}}\right. \\
& +\int_{0}^{+\infty} e^{y_{t_{i-1}, t_{i}}} p\left(y_{t_{i-1}, t_{i}}\right) d y_{t_{i-1}, t_{i}}-\int_{-\infty}^{0} e^{\left.y_{t_{i-1}, t_{i}} p\left(y_{t_{i-1}, t_{i}}\right) d y_{t_{i-1}, t_{i}}\right]}
\end{aligned}
$$

if $p\left(y_{t_{i-1}, t_{i}}\right)$ is the forward density function of $y_{t_{i-1}, t_{i}}$ under the forward measure $\mathbb{Q}^{T}$. With

$$
f\left(\phi, t, T ; S_{0}, v_{0}, r_{0}, X_{0}\right)=E^{Q^{T}}\left[e^{j \phi y_{t, T}} \mid S_{0}, v_{0}, r_{0}, X_{0}\right]
$$

representing the forward characteristic function of the underlying price under the forward measure $\mathbb{Q}^{\mathbb{T}}$, the delivery prices of a variance swap can be simplified as

$$
K_{\text {var }}=\frac{100^{2}}{T} \sum_{i=1}^{N}\left[f\left(-2 j, t_{i-1}, t_{i} ; S_{0}, v_{0}, r_{0}, X_{0}\right)-2 f\left(-j, t_{i-1}, t_{i} ; S_{0}, v_{0}, r_{0}, X_{0}\right)+1\right]
$$

and we can also obtain

$$
\int_{0}^{+\infty} p\left(y_{t_{i-1}, t_{i}}\right) d y_{t_{i-1}, t_{i}}=\frac{1}{2}+\frac{1}{\pi} \int_{0}^{+\infty} R E\left[\frac{f\left(\phi, t_{i-1}, t_{i} ; S_{0}, v_{0}, r_{0}, X_{0}\right)}{j \phi}\right] d \phi .
$$

Moreover, the definition of the forward characteristic function reveals that $\frac{e^{y t_{i-1}, t_{i}} p\left(y_{t_{i-1}, t_{i}}\right)}{f\left(-j, t_{i-1}, t_{i} ; S_{0}, v_{0}, r_{0}, X_{0}\right)}$ is the density of a certain random variable, whose forward characteristic function can be expressed as

$$
\bar{f}\left(\phi, t_{i-1}, t_{i} ; S_{0}, v_{0}, r_{0}, X_{0}\right)=\frac{f\left(\phi-j, t_{i-1}, t_{i} ; S_{0}, v_{0}, r_{0}, X_{0}\right)}{f\left(-j, t_{i-1}, t_{i} ; S_{0}, v_{0}, r_{0}, X_{0}\right)}
$$


In this sense,

$$
\begin{aligned}
\int_{0}^{+\infty} e^{y_{t_{i-1}, t_{i}} p\left(y_{t_{i-1}, t_{i}}\right) d y_{t_{i-1}, t_{i}}} & =f\left(-j, t_{i-1}, t_{i} ; S_{0}, v_{0}, r_{0}, X_{0}\right) \int_{0}^{+\infty} \frac{e^{y_{t_{i-1}, t_{i}} p\left(y_{t_{i-1}, t_{i}}\right)}}{f\left(-j, t_{i-1}, t_{i} ; S_{0}, v_{0}, r_{0}, X_{0}\right)} d y_{t_{i-1}, t_{i}} \\
& =\frac{1}{2}+\frac{1}{\pi} \int_{0}^{+\infty} R E\left[\frac{f\left(\phi-j, t_{i-1}, t_{i} ; S_{0}, v_{0}, r_{0}, X_{0}\right)}{j \phi \cdot f\left(-j, t_{i-1}, t_{i} ; S_{0}, v_{0}, r_{0}, X_{0}\right)}\right] d \phi
\end{aligned}
$$

from which the delivery price of a volatility swap can be arranged into

$$
K_{v o l}=100 \sqrt{\frac{2}{\pi N T}} \int_{0}^{+\infty} \sum_{i=1}^{N} R E\left[\frac{f\left(\phi-j, t_{i-1}, t_{i} ; S_{0}, v_{0}, r_{0}, X_{0}\right)-f\left(\phi, t_{i-1}, t_{i} ; S_{0}, v_{0}, r_{0}, X_{0}\right)}{j \phi}\right] d \phi .
$$

Although we have now expressed both delivery prices of variance and volatility swaps in terms of the forward characteristic function $f\left(\phi, t, T ; S_{0}, v_{0}, r_{0}, X_{0}\right),(3.3)$ and (3.4) are still not exact and analytical because the forward characteristic function remains unknown. Thus, in the next subsection, how to analytically derive the forward characteristic function will be illustrated.

\subsection{Forward characteristic function}

This subsection is devoted to deriving the forward characteristic function of the underlying price under the $T$-forward measure $\mathbb{Q}^{T}$, which can be achieved after conducting the measure transform as the dynamics of the underlying price we currently have, (2.2), are defined under the measure $\mathbb{Q}$.

If we denote

$$
\mu^{Q}=\left[\begin{array}{c}
r_{t} \\
k\left(\theta-v_{t}\right) \\
\alpha\left(\beta-r_{t}\right)
\end{array}\right], \Sigma=\left[\begin{array}{cccc}
\sqrt{v} & \xi_{X_{t}} & 0 & 0 \\
0 & 0 & \sigma \sqrt{v} & 0 \\
0 & 0 & 0 & \eta \sqrt{r}
\end{array}\right]
$$


and represent $C$ as

$$
C=\left[\begin{array}{cccc}
1 & 0 & 0 & 0 \\
0 & 1 & 0 & 0 \\
\rho & 0 & \sqrt{1-\rho^{2}} & 0 \\
0 & 0 & 0 & 1
\end{array}\right],
$$

System (2.2) has an alternative expression

$$
\left[\begin{array}{c}
\frac{d S_{t}}{S_{t}} \\
d v_{t} \\
d r_{t}
\end{array}\right]=\mu^{Q} d t+\Sigma \times C \times\left[\begin{array}{c}
d W_{1, t} \\
d W_{2, t} \\
d W_{3, t} \\
d W_{4, t}
\end{array}\right]
$$

where $W_{i, t}, i=1,2,3,4$ are four standard Brownian motions independent of each other. According to [4], the key step in making measure transform is to find the expression of the drift term $\mu^{Q^{T}}$ under the forward measure, which in fact requires the knowledge of the numeraires under both measures. In particular, the numeraires under under $\mathbb{Q}$ and $\mathbb{Q}^{T}$ are respectively $N_{1, t}=e^{\int_{0}^{t} r(s) d s}$ and $N_{2, t}=P(r, t, T)$, which is the $T$-maturity zero coupon bond price under $\mathbb{Q}$, with the formula [13]

$$
\begin{aligned}
P(r, t, T) & =e^{A(t, T)-B(t, T) r}, \\
A(t, T) & =-\alpha \beta\left\{\frac{4}{(m-\alpha)(m+\alpha)} \ln \left[\frac{2 m+(m+\alpha)\left(e^{m(T-t)}-1\right)}{2 m}\right]+\frac{2}{\alpha-m}(T-t)\right\}, \\
B(t, T) & =\frac{2\left(e^{m(T-t)}-1\right)}{2 m+(\alpha+m)\left(e^{m(T-t)}-1\right)},
\end{aligned}
$$

where $m=\sqrt{\alpha^{2}+2 \eta^{2}}$. Thus, the volatility terms of the two numeraires are denoted by 
$\sigma^{N_{1, t}}=(0,0,0,0)^{T}$ and $\sigma^{N_{2, t}}=\left(0,0,0,-\eta \sqrt{r} N_{2, t} B\right)^{T}$, respectively, leading to

$$
\mu^{Q_{T}}=\mu^{Q}-\Sigma \times \rho \times\left(\frac{\sigma^{N_{1, t}}}{N_{1, t}}-\frac{\sigma^{N_{2, t}}}{N_{2, t}}\right)=\left[\begin{array}{c}
r_{t} \\
k\left(\theta-v_{t}\right) \\
\alpha \beta-\left[\alpha+B \eta^{2}\right] r_{t}
\end{array}\right]
$$

with which the corresponding model dynamics under the forward measure $\mathbb{Q}^{T}$ can be obtained

$$
\left[\begin{array}{c}
\frac{d S_{t}}{S_{t}} \\
d v_{t} \\
d r_{t}
\end{array}\right]=\left[\begin{array}{c}
r_{t} \\
k\left(\theta-v_{t}\right) \\
\alpha \beta-\left[\alpha+B(t, T) \eta^{2}\right] r
\end{array}\right] d t+\Sigma \times C \times\left[\begin{array}{c}
d W_{1, t}^{Q^{T}} \\
d W_{2, t}^{Q^{T}} \\
d W_{3, t}^{Q^{T}} \\
d W_{4, t}^{Q^{T}}
\end{array}\right]
$$

We now alternatively express

$$
f\left(\phi, t, T ; S_{0}, v_{0}, r_{0}, X_{0}\right)=E^{Q^{T}}\left\{E^{Q^{T}}\left[e^{j \phi y_{t, T}} \mid S_{0}, v_{0}, r_{0}, X_{T}\right] \mid X_{0}\right\}
$$

from which we are actually treating the Markov chain as a deterministic process in the first step to compute the conditional forward characteristic function (the inner expectation)

$$
m\left(\phi, t, T ; S_{0}, v_{0}, r_{0} \mid X_{T}\right)=E^{Q^{T}}\left[e^{j \phi y_{t, T}} \mid S_{0}, v_{0}, r_{0}, X_{T}\right]
$$

However, as $y_{t, T}$ involves both $S_{t}$ and $S_{T}$, while we only have the information of the underlying price up to time 0 , we further represent $m\left(\phi, t, T ; S_{0}, v_{0}, r_{0} \mid X_{T}\right)$ in the form of

$$
\begin{aligned}
m\left(\phi, t, T ; S_{0}, v_{0}, r_{0} \mid X_{T}\right) & =E^{Q^{T}}\left\{E^{Q^{T}}\left[e^{j \phi y_{t}, T} \mid S_{t}, v_{t}, r_{t}, X_{T}\right] \mid S_{0}, v_{0}, r_{0}, X_{T}\right\} \\
& =E^{Q^{T}}\left\{e^{-j \phi z_{t}} h\left(\phi, t, T ; S_{t}, v_{t}, r_{t} \mid X_{T}\right) \mid S_{0}, v_{0}, r_{0}, X_{T}\right\}
\end{aligned}
$$


if we assume

$$
h\left(\phi, t, T ; S_{t}, v_{t}, r_{t} \mid X_{T}\right)=E^{Q^{T}}\left[e^{j \phi z_{T}} \mid S_{t}, v_{t}, r_{t}, X_{T}\right]
$$

as the conditional characteristic function. In this case, we need to firstly figure out $h\left(\phi, t, T ; S_{t}, v_{t}, r_{t} \mid X_{T}\right)$, the solution to which is presented in the following theorem.

Theorem 1 If the underlying price, volatility and the interest rate follow the dynamics (3.8), we have

$$
h\left(\phi, t, T ; S_{t}, v_{t}, r_{t} \mid X_{T}\right)=e^{C(\phi ; \tau)+D(\phi ; \tau) v_{t}+E(\phi ; \tau) r_{t}+j \phi z_{t}}, \tau=T-t
$$

where

$$
\begin{aligned}
D(\phi ; \tau) & =\frac{d-(j \phi \rho \sigma-k)}{\sigma^{2}} \cdot \frac{1-e^{d \tau}}{1-g e^{d \tau}}, \\
E(\phi ; \tau) & =-\frac{2 \sum_{n=0}^{+\infty}(n+1) \hat{a}_{n+1} \tau^{n}}{\eta^{2} \sum_{n=0}^{+\infty} \hat{a}_{n} \tau^{n}}, \\
C(\phi ; \tau) & =\tilde{C}(\phi ; \tau)+p(\phi ; \tau), \\
d & =\sqrt{(j \phi \rho \sigma-k)^{2}+\sigma^{2}\left(j \phi+\phi^{2}\right)}, g=\frac{(j \phi \rho \sigma-k)-d}{(j \phi \rho \sigma-k)+d}, \\
\hat{a}_{n+2} & =-\frac{\hat{I}}{2 m(n+1)(n+2)}, n \geq 0, \hat{a}_{0}=1, \hat{a}_{1}=0, \\
\hat{I} & =2 \alpha m(n+1) \hat{a}_{n+1}+j \phi \eta^{2} m \hat{a}_{n}+(\alpha+m) \sum_{i=1}^{n}(n+2-i)(n+1-i) c_{i} \hat{a}_{n+2-i} \\
& +\left(\alpha^{2}+\alpha m+2 \eta^{2}\right) \sum_{i=1}^{n}(n+1-i) c_{i} \hat{a}_{n+1-i}+\frac{1}{2} j \phi \eta^{2}(\alpha+m) \sum_{i=1}^{n} c_{i} \hat{a}_{n-i}, c_{i}=\frac{m^{n}}{n !} \\
\tilde{C}(\phi ; \tau) & =\frac{k \theta}{\sigma^{2}}\left\{[d-(j \phi \rho \sigma-k)] \tau-2 \ln \left(\frac{1-g e^{d \tau}}{1-g}\right)\right\}-\alpha \beta \int_{0}^{\tau} \frac{2 \sum_{n=0}^{+\infty}(n+1) \hat{a}_{n+1} t^{n}}{\eta^{2} \sum_{n=0}^{+\infty} \hat{a}_{n} t^{n}} d t, \\
p(\phi ; \tau) & =-\frac{1}{2}\left(j \phi+\phi^{2}\right) \int_{t}^{T}<\bar{\xi}, X_{s}>d s .
\end{aligned}
$$

Proof. From the definition of $h\left(\phi, t, T ; S_{t}, v_{t}, r_{t} \mid X_{T}\right)$, it is not difficult to find that it 
satisfies the following PDE (partial differential equation) system

$$
\begin{aligned}
\frac{\partial h}{\partial \tau} & =\frac{1}{2}\left(v+\xi_{t}^{2}\right) \frac{\partial^{2} h}{\partial z^{2}}+\frac{1}{2} \sigma^{2} v \frac{\partial^{2} h}{\partial v^{2}}+\frac{1}{2} \eta^{2} r \frac{\partial^{2} h}{\partial r^{2}}+\rho \sigma v \frac{\partial^{2} h}{\partial y \partial v}+\left[r-\frac{1}{2}\left(v+\xi_{t}^{2}\right)\right] \frac{\partial h}{\partial y} \\
& +k(\theta-v) \frac{\partial h}{\partial v}+\left\{\alpha \beta-\left[\alpha+B(s, T) \eta^{2}\right] r\right\} \frac{\partial h}{\partial r} \\
\left.h\right|_{\tau=0} & =e^{j \phi y_{t, T}} .
\end{aligned}
$$

This PDE system can be transformed into three ODE (ordinary differential equation) systems

$$
\begin{aligned}
& \frac{d D}{d \tau}=\frac{1}{2} \sigma^{2} D^{2}+(j \phi \rho \sigma-k) D-\frac{1}{2}\left(j \phi+\phi^{2}\right), D(\phi ; 0)=0, \\
& \frac{d E}{d \tau}=\frac{1}{2} \eta^{2} E^{2}-\left[\alpha+B(s, T) \eta^{2}\right] E+j \phi, E(\phi ; 0)=0, \\
& \frac{d C}{d \tau}=k \theta D+\alpha \beta E-\frac{1}{2}\left(j \phi+\phi^{2}\right) \xi_{t}^{2}, C(\phi ; 0)=0,
\end{aligned}
$$

with the substitution of the specific form of $h\left(\phi, t, T ; S_{t}, v_{t}, r_{t} \mid X_{T}\right)$ presented in (3.12). To get the expression of $D(\phi ; \tau)$, one only need to solve a Riccati equation with constant coefficients, which can be achieved without much effort. However, $E(\phi ; \tau)$ is more difficult to be obtained as the coefficients of the ODE are no longer constant. As usual, after applying the transform of

$$
E(\phi ; \tau)=-\frac{2 u^{\prime}(\tau)}{\eta^{2} u(\tau)}
$$

to turn the Riccati equation into a second order linear ODE

$$
u^{\prime \prime}+\left[\alpha+B(s, T) \eta^{2}\right] u^{\prime}+\frac{1}{2} j \phi \eta^{2}=0,
$$

we actually try to express the solution to $u(\tau)$ in the series form

$$
u=\sum_{n=0}^{+\infty} a_{n} \tau^{n}
$$

with the coefficients of $a_{n}, n \geq 0$ to be determined. With the expansion of $e^{m \tau}=\sum_{n=0}^{+\infty} c_{n} \tau^{n}$ 
and some algebraic calculations, it is straightforward that

$$
\sum_{n=0}^{+\infty} b_{n} \tau^{n}=0
$$

where

$$
\begin{aligned}
b_{n} & =2 m(n+1)(n+2) a_{n+2}+2 \alpha m(n+1) a_{n+1}+j \phi \eta^{2} m a_{n}+(\alpha+m) \sum_{i=1}^{n}(n+2-i)(n+1-i) c_{i} a_{n+2-i} \\
& +\left(\alpha^{2}+\alpha m+2 \eta^{2}\right) \sum_{i=1}^{n}(n+1-i) c_{i} a_{n+1-i}+\frac{1}{2} j \phi \eta^{2}(\alpha+m) \sum_{i=1}^{n} c_{i} a_{n-i}, n \geq 0
\end{aligned}
$$

Clearly, $b_{n}, n \geq 0$ should all be zero, which yields the recurrence relationship for $a_{n}$, with $a_{0}$ and $a_{1}$ as the required initial values. However, due to the lack of the value for $a_{0}\left(a_{1}=0\right.$ can be easily derived from $E(\phi ; 0)=0)$, we divide both sides of (3.16), with the notation $\hat{b}_{n}=\frac{b_{n}}{a_{0}}$ and $\hat{a}_{n}=\frac{a_{n}}{a_{0}}$. In this case, $\hat{b}_{n}=0, n \geq 0$ leads to the recurrence relationship for $\hat{a}_{n}$ combined with $\hat{a}_{0}=1$ and $\hat{a}_{1}=0$, from which one can obtain the formula for $E(\phi ; \tau)^{2}$. Finally, by noticing the fact that $\xi_{t}$ is only a deterministic function of the time, direct integration of its ODE produces the desired result. This has completed the proof.

Substituting the expression of $h\left(\phi, t, T ; S_{t}, v_{t}, r_{t} \mid X_{T}\right),(3.12)$, into (3.10) gives

$$
m\left(\phi, t, T ; v_{0}, r_{0} \mid X_{T}\right)=e^{C(\phi ; \tau)} E^{Q^{T}}\left[e^{D(\phi ; \tau) v_{t}+E(\phi ; \tau) r_{t}} \mid v_{0}, r_{0}, X_{T}\right]
$$

which no longer depends on the underlying price as a result of canceling $z_{t}$ and is formulated in Theorem 2 .

Theorem 2 If the underlying price, volatility and the interest rate follow the dynamics

\footnotetext{
${ }^{2}$ Although it happens that the time-dependent Riccati equation for $E(\phi ; \tau)$ here could be analytically solved with symbolic calculations using some software like Maple, the adopted series solution technique is still valuable as time-dependent Riccati equations usually do not admit such kind of analytical solutions, and the series solution technique can be extended to solve those cases.
} 
(3.8), the conditional forward characteristic function can be expressed as

$$
m\left(\phi, t, T ; v_{0}, r_{0} \mid X_{T}\right)=e^{C(\phi ; \tau)+\bar{C}(\phi ; t)+\bar{D}(\phi ; t) v_{0}+\bar{E}(\phi ; t) r_{0}}
$$

where

$$
\begin{aligned}
\bar{D}(\phi ; t) & =\frac{2 k}{\sigma^{2}} \frac{1}{1-\left[1-\frac{2 k}{\sigma^{2} D(\phi ; \tau)}\right] e^{k t_{s}}}, \\
\bar{E}(\phi ; t) & =\frac{e^{-\left(\alpha+\frac{2 \eta^{2}}{\alpha-m}\right) t} q(t)}{-\frac{1}{2} \eta^{2} \int_{0}^{t} e^{-\left(\alpha+\frac{2 \eta^{2}}{\alpha-m}\right) x} q(x) d x+\frac{1}{E(\phi ; \tau)}}, \\
\bar{C}(\phi ; t) & =\frac{2 k \theta}{\sigma^{2}}\left\{k t-\ln \left[1-\left(1-\frac{2 k}{\sigma^{2} D(\phi ; \tau)}\right) e^{k t}\right]+\ln \left[\frac{2 k}{\sigma^{2} D(\phi ; \tau)}\right]\right\}+\alpha \beta \int_{0}^{t} \bar{E}(\phi ; s) d s, \\
q(t) & =\left\{\frac{2 m+(m+\alpha)\left[e^{m(\tau+t)}-1\right]}{2 m+(m+\alpha)\left(e^{m \tau}-1\right)}\right\}^{-\frac{4 \eta^{2}}{(m-\alpha)(m+\alpha)}} .
\end{aligned}
$$

Proof. The PDE system governing

$$
w\left(\phi, s, t, T ; v_{s}, r_{s} \mid X_{T}\right)=E^{Q^{T}}\left\{e^{D(\phi ; \tau) v_{t}+E(\phi ; \tau) r_{t}} \mid v_{s}, r_{s}, X_{T}\right\}
$$

can be easily derived as

$$
\begin{aligned}
\frac{\partial w}{\partial \tau_{s}} & =\frac{1}{2} \sigma^{2} v \frac{\partial^{2} w}{\partial v^{2}}+\frac{1}{2} \eta^{2} r \frac{\partial^{2} w}{\partial r^{2}}+k(\theta-v) \frac{\partial w}{\partial v}+\{\alpha \beta-[\alpha+B(s, T)] r\} \frac{\partial w}{\partial r} \\
\left.w\right|_{\tau_{s}=0} & =e^{D(\phi ; \tau) v_{t}+E(\phi ; \tau) r_{t}}, \quad \tau_{s}=t-s .
\end{aligned}
$$

By assuming that

$$
w\left(\phi, s, t, T ; v_{s}, r_{s} \mid X_{T}\right)=e^{\bar{C}\left(\phi ; \tau_{s}\right)+\bar{D}\left(\phi ; \tau_{s}\right) v_{s}+\bar{E}\left(\phi ; \tau_{s}\right) r_{s}}
$$


three new ODE systems for $\bar{D}\left(\phi ; \tau_{s}\right), \bar{E}\left(\phi ; \tau_{s}\right)$ and $\bar{C}\left(\phi ; \tau_{s}\right)$ can be specified as

$$
\begin{aligned}
& \frac{d \bar{D}}{d \tau_{s}}=\frac{1}{2} \sigma^{2} \bar{D}^{2}-k \bar{D}, D(\phi ; 0)=D(\phi ; \tau), \\
& \frac{d \bar{E}}{d \tau_{s}}=\frac{1}{2} \eta^{2} \bar{E}^{2}-\left[\alpha+B(s, T) \eta^{2}\right] E, E(\phi ; 0)=E(\phi ; \tau), \\
& \frac{d \bar{C}}{d \tau_{s}}=k \theta \bar{D}+\alpha \beta \bar{E}, C(\phi ; 0)=0 .
\end{aligned}
$$

The ODEs governing for $\bar{D}\left(\phi ; \tau_{s}\right)$ and $\bar{E}\left(\phi ; \tau_{s}\right)$ are both Bernoulli's equation, implying that the ODEs for $\frac{1}{\bar{D}\left(\phi ; \tau_{s}\right)}$ and $\frac{1}{\bar{E}\left(\phi ; \tau_{s}\right)}$ are both first order linear ODEs, which can be easily solved. With the expressions of $\bar{D}\left(\phi ; \tau_{s}\right)$ and $\bar{E}\left(\phi ; \tau_{s}\right)$, the formula of $\bar{C}\left(\phi ; t_{s}\right)$ can be found through direct integration. Considering that

$$
m\left(\phi, t, T ; v_{0}, r_{0} \mid X_{T}\right)=e^{C(\phi ; \tau)} E^{Q^{T}}\left[w\left(\phi, 0, t, T ; v_{0}, r_{0} \mid X_{T}\right) \mid v_{0}, r_{0}, X_{T}\right]
$$

we have already completed the proof.

The target forward characteristic function is nothing but the expectation of the conditional characteristic function

$$
\begin{aligned}
f\left(\phi, t, T ; v_{0}, r_{0}, X_{0}\right) & =E^{Q^{T}}\left[m\left(\phi, t, T ; v_{0}, r_{0} \mid X_{T}\right) \mid X_{0}\right] \\
& =e^{\tilde{C}(\phi ; \tau)+\bar{C}(\phi ; t)+\bar{D}(\phi ; t) v_{0}+\bar{E}(\phi ; t) r_{0}} E^{Q^{T}}\left[e^{p(\phi ; \tau)} \mid X_{0}\right]
\end{aligned}
$$

the solution to which is provided in Theorem 3.

Theorem 3 If the underlying price, volatility and the interest rate follow the dynamics (3.8), the forward characteristic function can be worked out as

$$
f\left(\phi, t, T ; v_{0}, r_{0}, X_{0}\right)=e^{\tilde{C}(\phi ; \tau)+\bar{C}(\phi ; t)+\bar{D}(\phi ; t) v_{0}+\bar{E}(\phi ; t) r_{0}}<P b, X_{0}>
$$


where

$$
\begin{aligned}
P & =\left(\begin{array}{ll}
p_{11}(t) & p_{12}(t) \\
p_{21}(t) & p_{22}(t)
\end{array}\right), b=\left(\begin{array}{c}
<e^{A^{T} \tau+B} X_{1}, I> \\
<e^{A^{T} \tau+B} X_{2}, I>
\end{array}\right), I=(1,1)^{T}, \\
A & =\left(\begin{array}{cc}
-\lambda_{12} & \lambda_{12} \\
\lambda_{21} & -\lambda_{21}
\end{array}\right), B=\left(\begin{array}{cc}
-\frac{1}{2}\left(j \phi+\phi^{2}\right) \xi_{1}^{2} \tau & 0 \\
0 & -\frac{1}{2}\left(j \phi+\phi^{2}\right) \xi_{2}^{2} \tau
\end{array}\right), \\
p_{11}(t) & =\frac{\lambda_{21}}{\lambda_{12}+\lambda_{21}}+\frac{\lambda_{12}}{\lambda_{12}+\lambda_{21}} e^{-\left(\lambda_{12}+\lambda_{21}\right) t}, p_{12}(t)=1-p_{11}(t), \\
p_{22}(t) & =\frac{\lambda_{12}}{\lambda_{12}+\lambda_{21}}+\frac{\lambda_{21}}{\lambda_{12}+\lambda_{21}} e^{-\left(\lambda_{12}+\lambda_{21}\right) t}, p_{21}(t)=1-p_{22}(t) .
\end{aligned}
$$

Proof. The derivation of $f\left(\phi, t, T ; v_{0}, r_{0}, X_{0}\right)$ requires the knowledge of the unknown expectation, $E^{Q^{T}}\left[e^{p(\phi ; \tau)} \mid X_{0}\right]$, in (3.21). If we rewrite it as

$$
\left.E^{Q^{T}}\left[e^{p(\phi ; \tau)} \mid X_{0}\right]=E^{Q^{T}}\left\{E^{Q^{T}}\left[e^{p(\phi ; \tau)} \mid X_{t}\right] \mid X_{0}\right]\right\}
$$

it is not difficult to derive the inner expectation

$$
E^{Q^{T}}\left[e^{p(\phi ; \tau)} \mid X_{t}\right]=<e^{A^{T} \tau+B} X_{t}, I>
$$

according to [7]. Since the inner expectation only involves the information of $X_{t}$, we can certainly obtain

$$
E^{Q^{T}}\left[<e^{A^{T} \tau+B} X_{t}, I>\mid X_{0}\right]=<P b, X_{0}>
$$

if we assume that $p_{i j}(t), i=1,2, j=1,2$ denote the probability of the Markov chain staying in State $j$ at time $t$ with $i$ being its initial state at time 0. This has completed the proof.

With the forward characteristic function being successfully derived, we are now able to price variance and volatility swaps using (3.3) and (3.4) respectively. However, one should notice that the solution is written in a series form, which is not safe to use unless it is accompanied with a convergence proof. Although it seems to be impossible to provide any 
proof of convergence, given the convoluted expressions of the formulae, we still manage to provide a radius of convergence for our pricing formulae, the details of which are provided in the following theorem.

Theorem 4 The variance and volatility swap pricing formulae, (3.3) and (3.4), will always converge if

$$
\tau \leq \frac{1}{m} \sqrt{\left[\ln \left(\frac{m-\alpha}{m+\alpha}\right)\right]^{2}+\pi^{2}}
$$

Proof. The proof is quite straightforward, if one makes use of the theory regarding the convergence of the series solution to second order linear ODEs [2], as the only place where the series solution is introduced is in the procedure of solving Equation (3.14). The coefficients of the ODE are analytic in the entire complex domain except when

$$
2 m+(\alpha+m)\left(e^{m \tau_{s}}-1\right)=0
$$

implying that all the singularities are $\frac{1}{m} \ln \left(\frac{m-\alpha}{m+\alpha}\right)+j \frac{(2 k+1) \pi}{m}, k=0,1,2 \ldots$ Considering that $\tau=0$ is an ordinary point, the radius of convergence is at least the distance between zero and its nearest singularity $\frac{1}{m} \ln \left(\frac{m-\alpha}{m+\alpha}\right)+j \frac{\pi}{m}$; finding such a distance would certainly yield the desired result. This has completed the proof ${ }^{3}$.

With all the discussions above, it is not difficult to find that both pricing formulae for variance and volatility swaps, (3.3) and (3.4), are completely analytical. However, there are still several important issues to be addressed. Firstly, although the convergence of the solution is guaranteed, we are not clear about the speed of convergence, an important factor in practical implementation. Of course, the accuracy of the formulae should also be demonstrated to remove the possibility of algebraic errors contained in the derivation process. Finally, one may also be interested in the effect of the newly introduced factor on

\footnotetext{
${ }^{3}$ In cases where the radius of convergence is not satisfied, one can also follow a similar procedure presented in [13] to derive a set of pricing formulae so that one can always find a convergent formula for any time to expiry.
} 
variance and volatility swap prices, which could also provide some guidance for practical purposes. These will be illustrated in the next section.

\section{Numerical experiments and examples}

In this section, the speed of convergence as well as the accuracy of the derived formulae will be numerically checked first, and we will only use variance swap prices as an example since both formulae are established based on the forward characteristic function. After gaining confidence in our formulae, the influence of the regime switching is analyzed by comparing swap prices calculated under our model and those obtained from the Heston-CIR model. In the following, unless otherwise stated, both transition rates, $\lambda_{12}$ and $\lambda_{21}$, are set to 10 , while the values of the regime switching factor in both states, $\xi_{1}$ and $\xi_{2}$, are chosen as 0.1 and 0.2 , respectively, with the current state being 1 . The values of other parameters include $T=1, k=5, \theta=0.1, \sigma=0.1, \rho=-0.5, \alpha=5, \beta=0.1, \eta=0.1, v_{0}=r_{0}=0.03, N=4$, which are also used for the corresponding parameters of the Heston-CIR hybrid model for comparison purposes.

To demonstrate the speed of convergence, we display the difference between two subsequent terms against the number of terms used in the series solution in Figure 1(a). One can clearly observe that such a difference decreases very sharply to zero, the speed of which is very similar to each other when we change the expiry time. The closeness of 9-term and 10-term prices is further demonstrated in Figure 1(b), with maximum absolute difference between the two price being less than $10^{-4}$. These can lead to the conclusion that our solution converges very rapidly, and only a few terms will suffice to obtain accurate results. In this sense, the following numerical examples are produced with 10 terms taken in the series solution.

The quick speed of convergence satisfies practical demands due to the time intensiveness of the model calibration process, but the pricing formulae are still not safe the market 


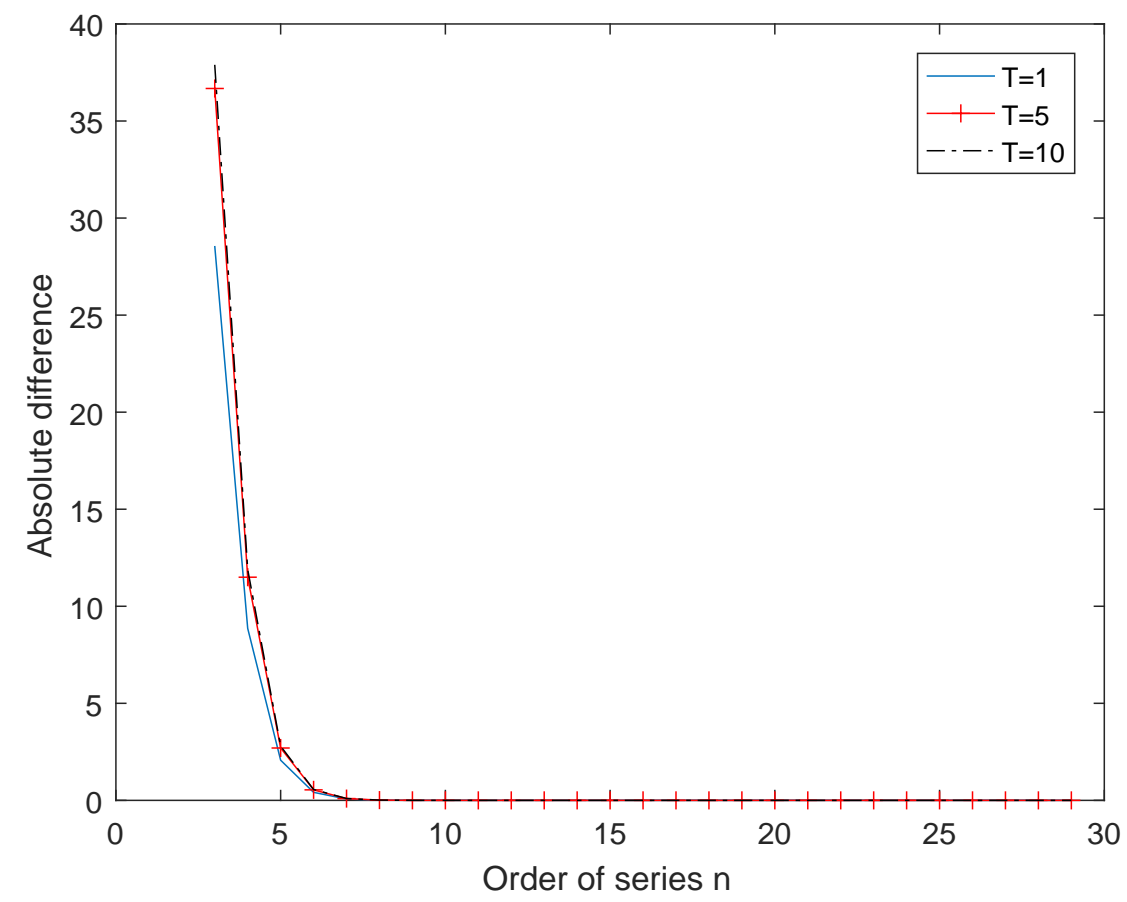

(a) Absolute difference between $(n+1)$-term and $n$-term variance swap price.

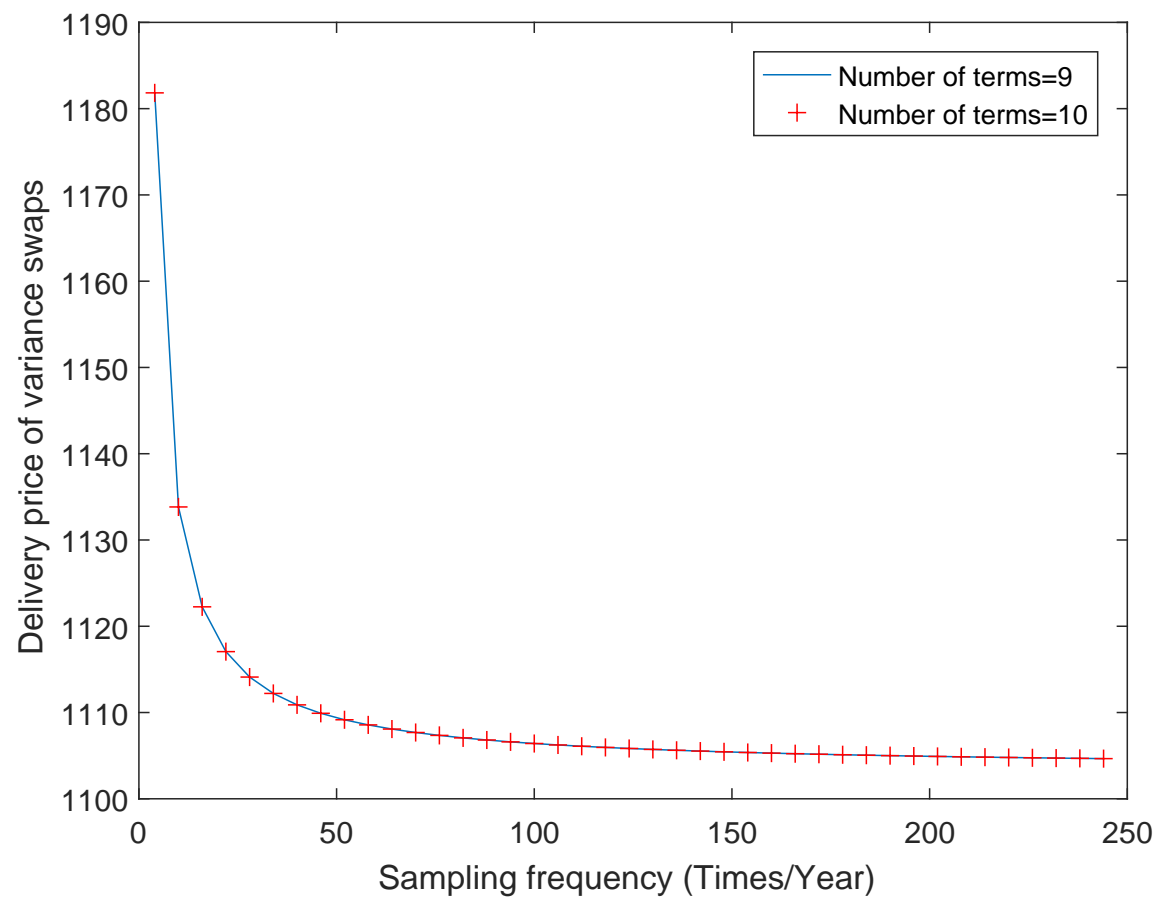

(b) The 10-term and 11-term variance swap prices.

Figure 1: Speed of convergence for our formula. 


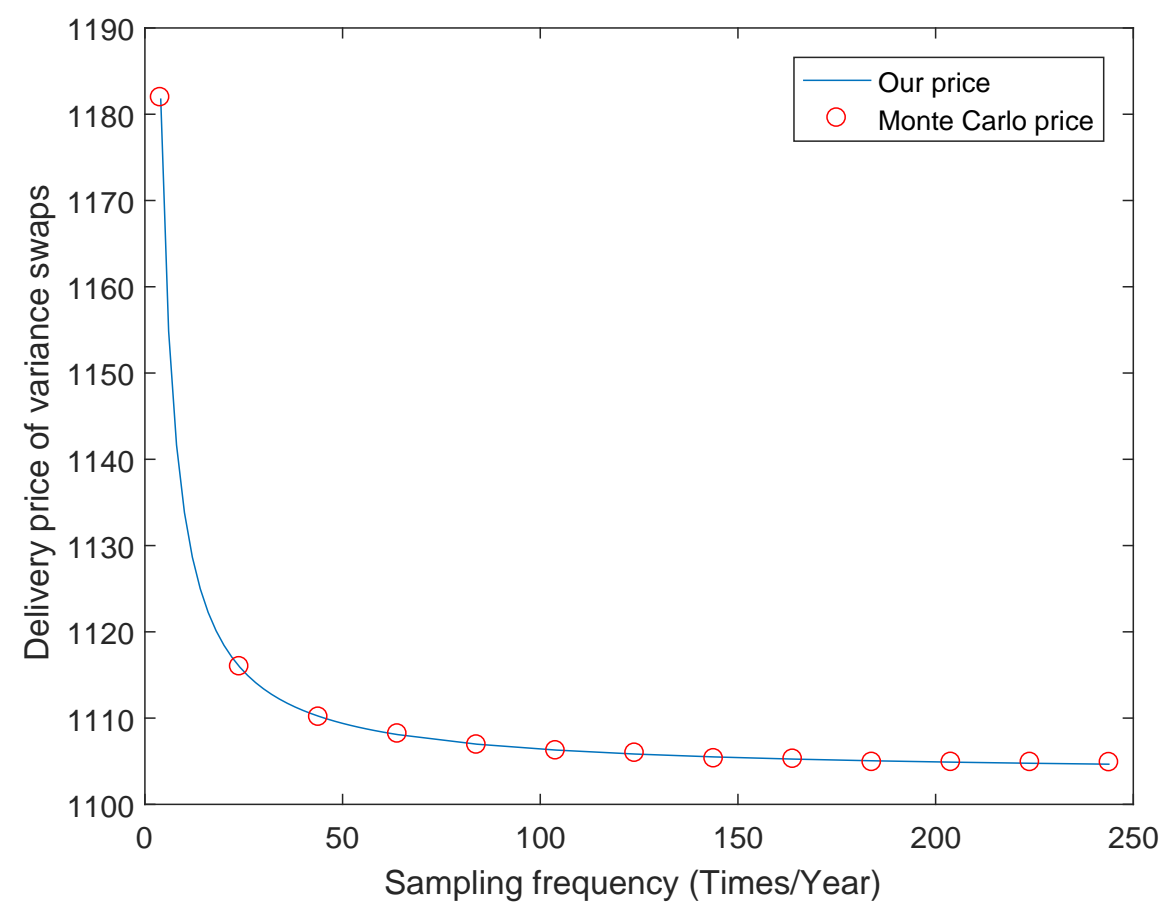

(a) Variance swap prices calculated from our formula and Monte Carlo simulation.

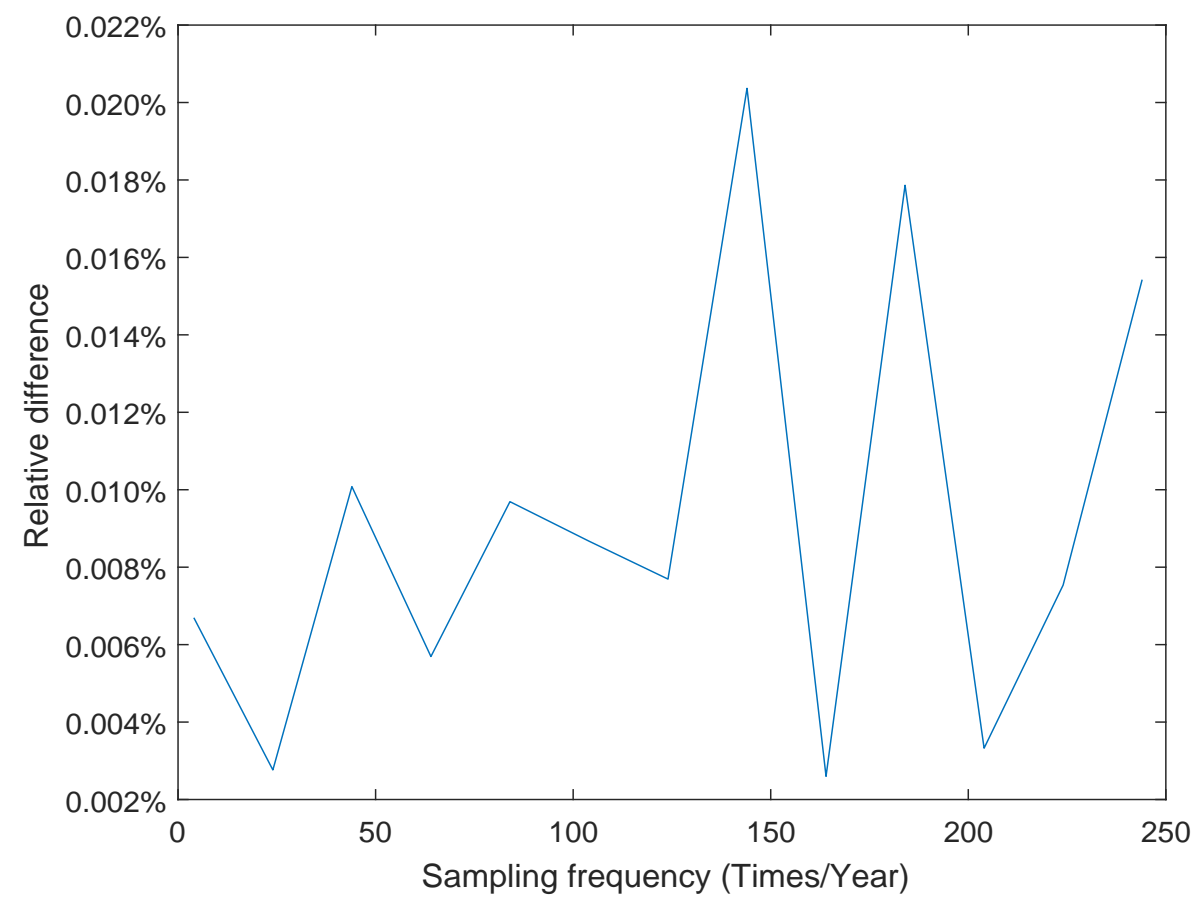

(b) Relative difference between our price and Monte Carlo price.

Figure 2: Our price vs Monte Carlo price. 
implementation, since there is no guarantee that the variance and volatility swap prices produced by them are indeed the ones under our model. To check the validity of the newly derived formulae, our prices (variance swap prices calculated through our formula) are benchmarked with Monte-Carlo prices (swap prices obtained through Monte Carlo simulation), as shown in Figure 2. The point-wise closeness between our price and MonteCarlo price in Figure 2(a) is a clear sign of the accuracy of our formula, and Figure 2(b) further verifies the formula by showing the relative difference between the two prices. The maximum relative difference being less than $0.025 \%$ can of course act as an evidence of the correctness of the formula.

With no doubt about the newly derived formulae, we are now able to use them to investigate the effect of the second regime switching factor on variance and volatility swap prices. To achieve this, we introduce a scale parameter $z$ varying within $[0,1]$, with which the two state values of the second factor are set to $\xi_{1}=0.1 * z$ and $\xi_{2}=0.2 * z$, and the corresponding results are presented in Figure 3. What can be observed first is that when the scale parameter is equal to zero, our prices of both states are nothing but the swap prices under the Heston-CIR hybrid model, which is expected since in this case the second factor indeed disappears. With the increase in the scale parameter, our prices of both states keep increasing, and they are always higher than the Heston-CIR price. This is financially meaningful since the larger the scale parameter, the greater of state values of the second factor, leading to a higher volatility and thus a higher delivery price. It is also interesting to find that our price of State 2 is always higher than that of State 1, which is a result of the second factor of State 1 is less than that of State 2, potentially leading a lower volatility when the underlying price stays in State 1 at the current time.

As the Heston-CIR model is a special case of our model, it is of interest to see the performance of both models when the degeneration does not occur. Therefore, variance and volatility swap prices under our model are compare with those from the Heston-CIR model in Figure 4, and one can easily observe that our prices of both states are higher than 


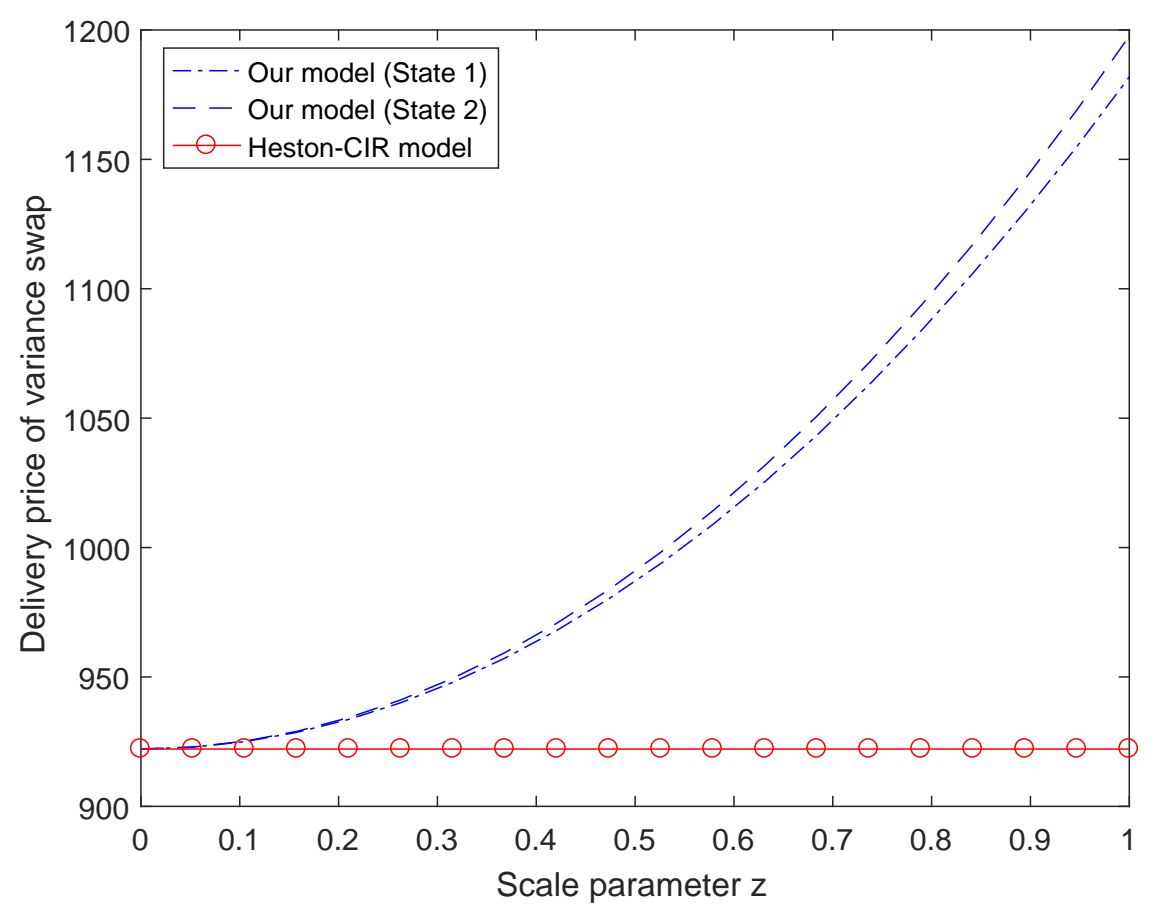

(a) Variance swap prices with respect to the scale parameter.

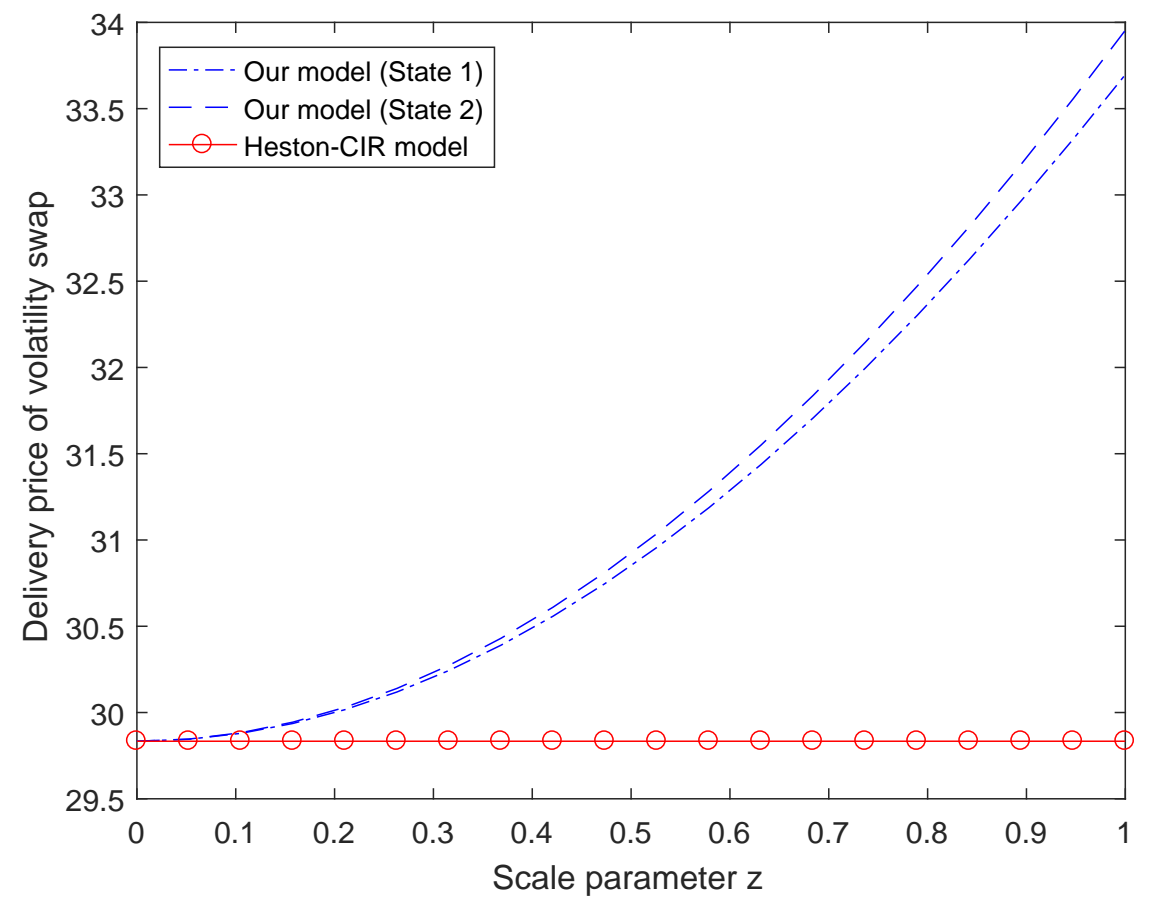

(b) Volatility swap prices with respect to the scale parameter.

Figure 3: The relationship between our price and Heston-CIR price. 


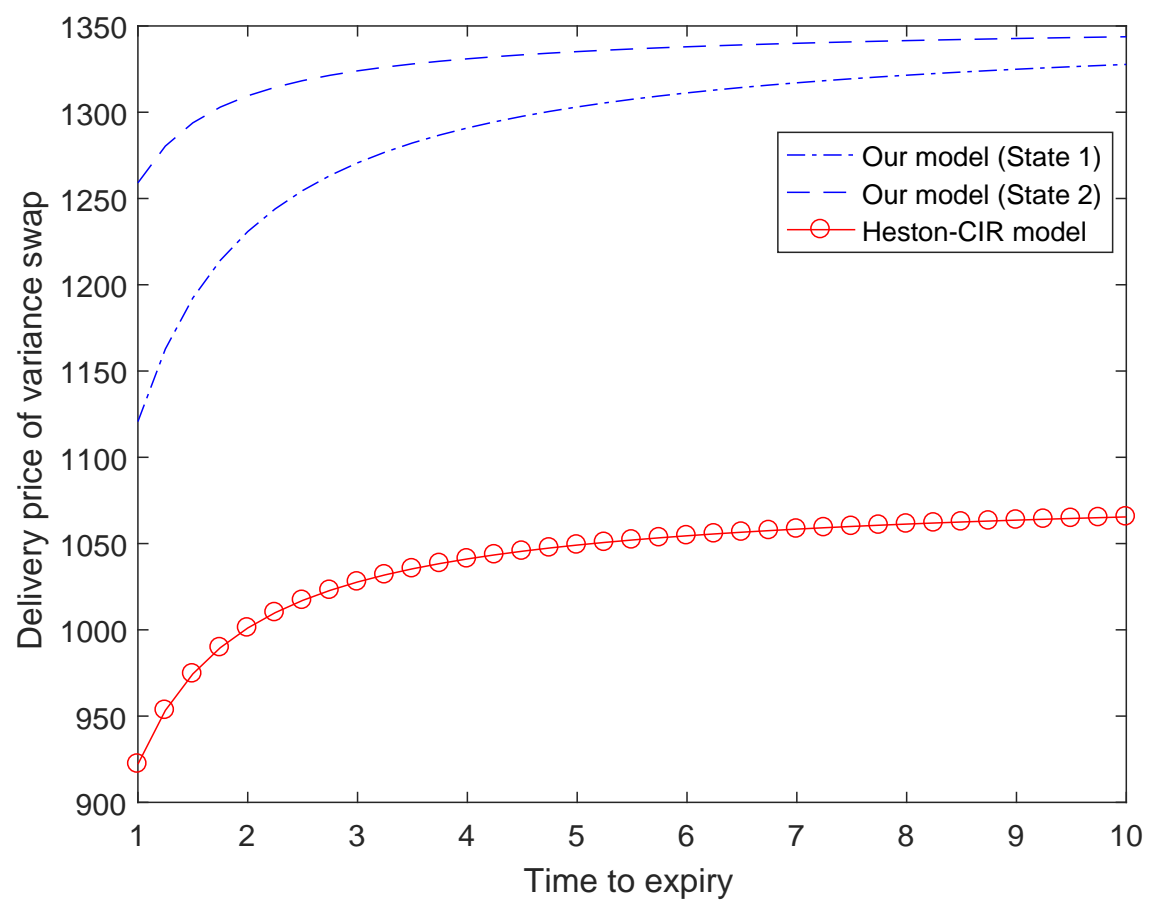

(a) Variance swap prices with respect to the expiry time.

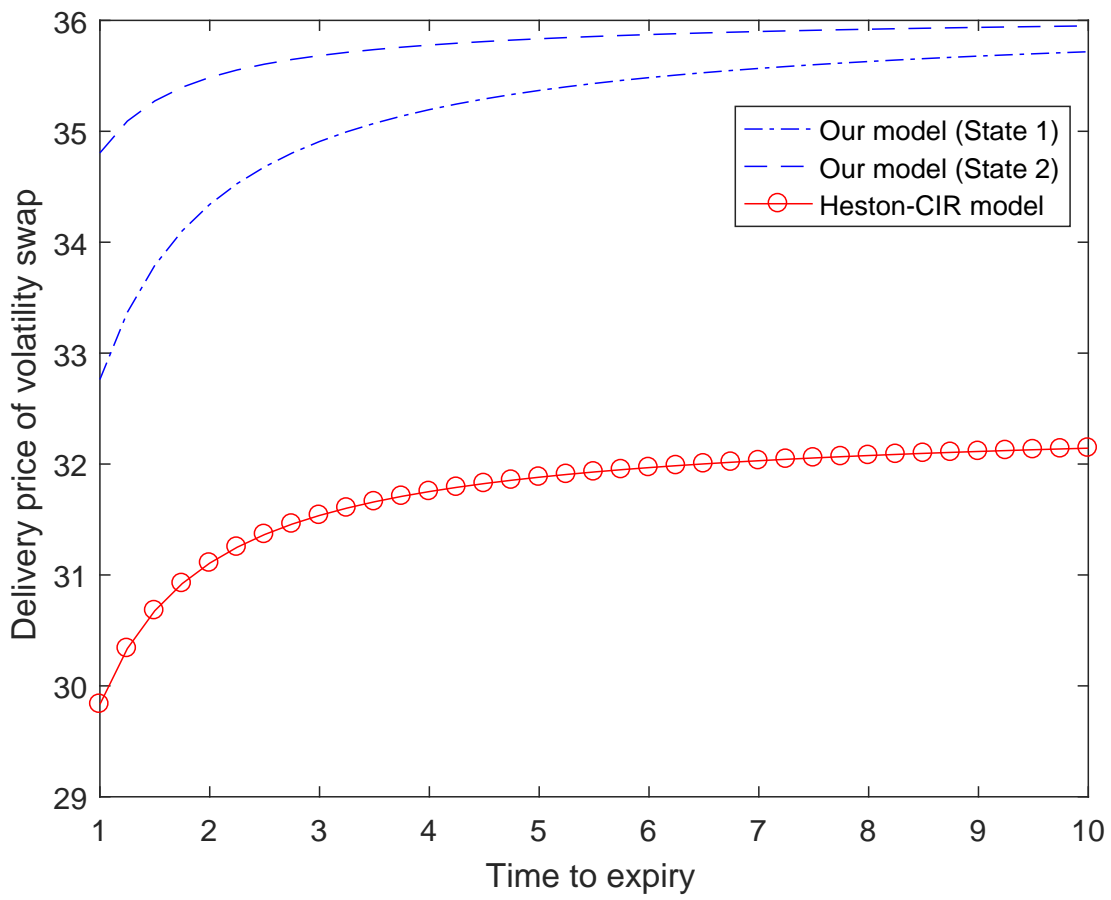

(b) Volatility swap prices with respect to the expiry time.

Figure 4: Our price vs Heston-CIR price. 
the corresponding Heston-CIR price, which is expected since when the regime switching factor is non-zero, the underlying price in our model has a higher level of volatility, which implies higher uncertainly, leading to a higher delivery price. However, this does not mean that our model can only be used in the cases where the Heston-CIR model is shown to underprice the variance and volatility swap contracts in real markets. This is because results obtained here are based on the fact that the corresponding parameters in both models are assumed to be the same, while this may not be the case when both models are used in practice, since model parameters will always be determined using real market data. In this sense, it is possible that the prices produced by our model are lower than those from the Heston model, using the estimated parameters.

Depicted in Figure 5 is the effect of the transition rates on variance and volatility swaps, when the two transition rates are assumed to be equal to each other. One can clearly observe distinct trend of swap prices corresponding to different states; while swap prices of State 1 are an increasing function of the transition rate, those of State 2 display a downward trend. This can be explained from the point of view that a larger transition rate could naturally weaken the effect of the initial state as it yields a higher probability for the underlying starting in State 1 (2) to switch to another state, leading to the increase (decrease) in the volatility and the swap prices.

It should be remarked that any mathematical model needs to go through a calibration process before it can be applied in practice, and thus it is very natural for us to consider the calibration of our two-factor Heston-CIR hybrid model. However, variance and volatility swaps are mainly over-the-counter derivatives and thus collecting their market data is never as easy as acquiring data of exchange-traded derivatives. Nevertheless, one should never devalue our theoretical work here, as the analytical pricing formulae derived in this paper can facilitate the calibration process for those practitioners who have access to the data.

Of course, model calibration involving regime switching is different from that without regime switching as it is very difficult to determine which state price should be regarded 


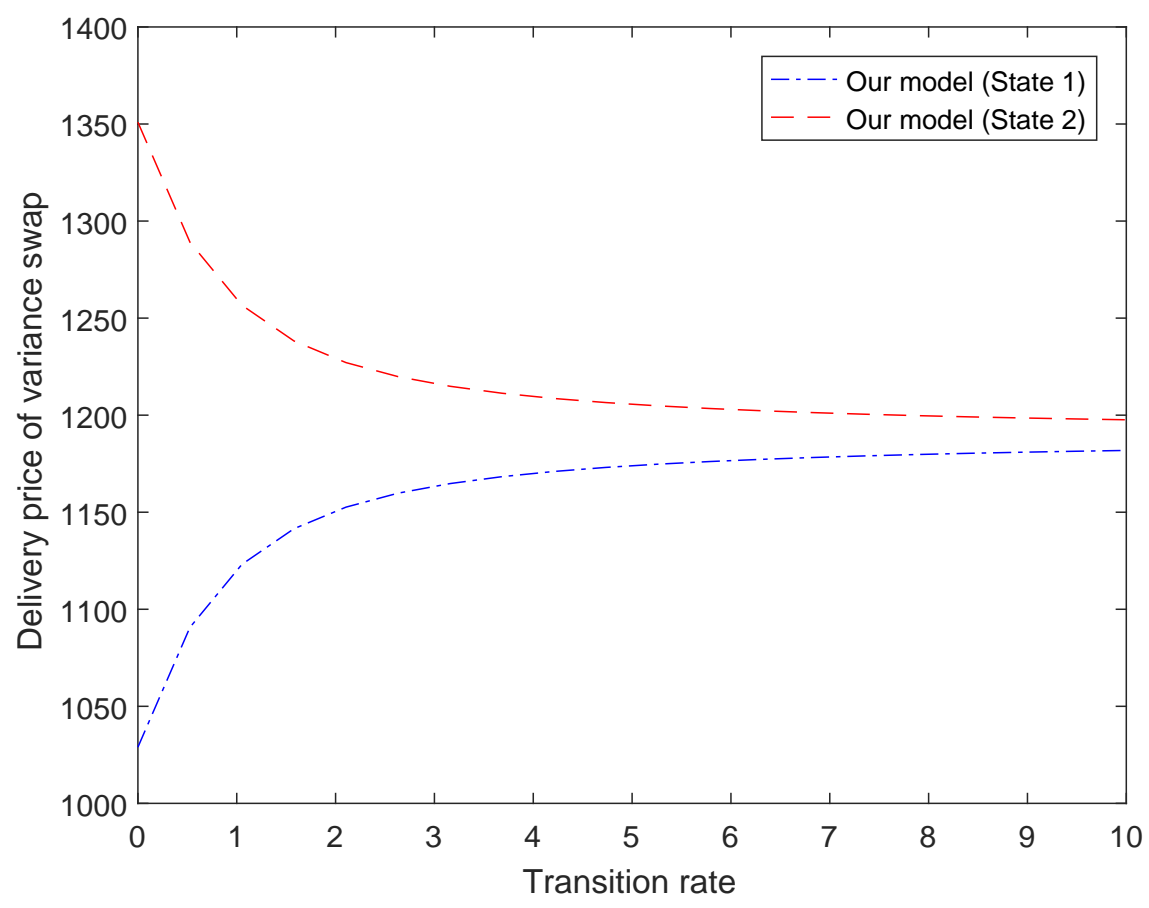

(a) Variance swap prices with respect to the transition rates.

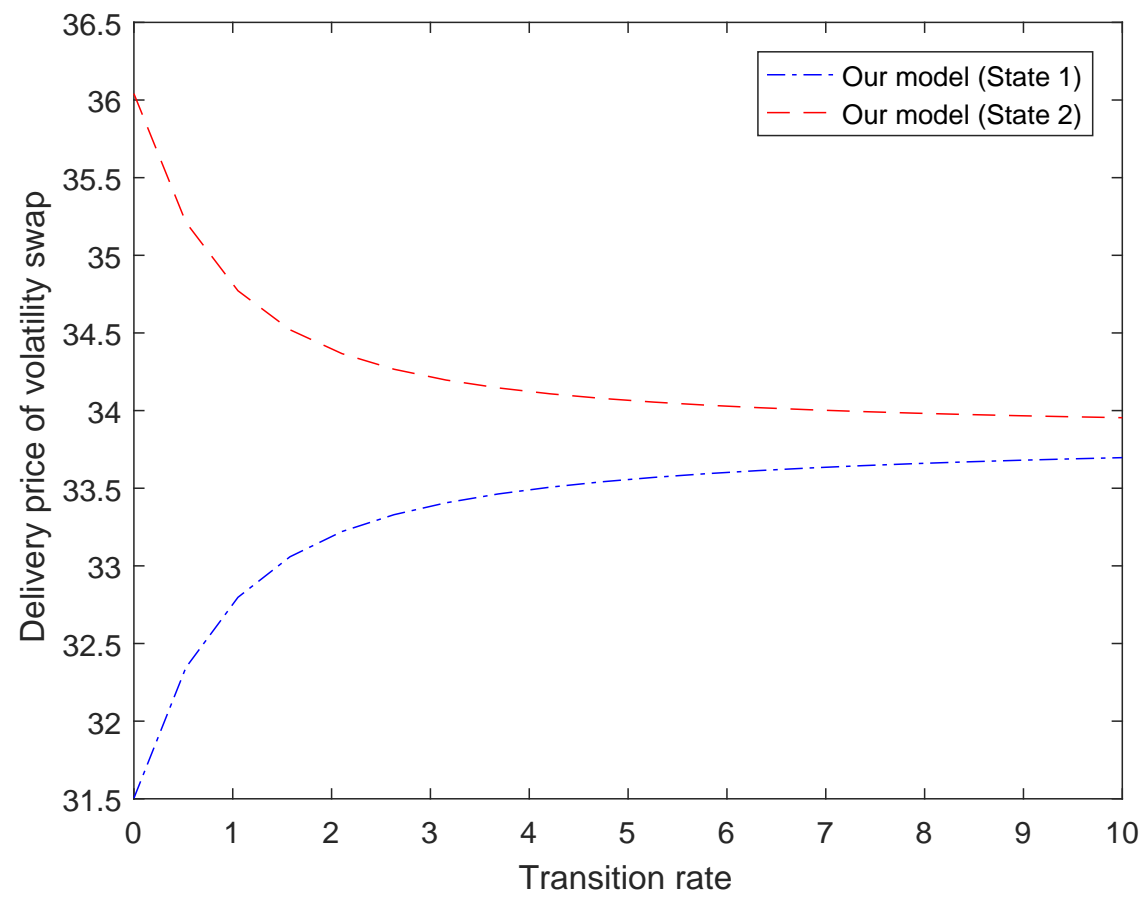

(b) Volatility swap prices with respect to the transition rates.

Figure 5: The effect of the transition rates. 
as the model price, given that we usually do not have the knowledge of the state the underlying asset price belongs to in practice. Fortunately, this problem has been resolved by the new closed system proposed by He \& Zhu [12], and the calibration of our model can be easily achieved following a similar procedure with our newly derived formulae.

\section{Conclusion}

In this paper, the Heston stochastic volatility, CIR stochastic interest rate and regime switching are combined together to formulate a two-factor Heston-CIR hybrid model. This model still possesses analytical tractability for variance and volatility swap prices, which is achieved after the successful derivation of the forward characteristic function. The obtained analytical pricing formulae are in in fact series solutions, the convergence of which is guaranteed with a radius of convergence. Through numerical experiments, the rapid speed of convergence, the accuracy of the newly derived formulae as well as the significant impact of the newly introduced regime switching factor demonstrate the potential of the formulae to be applied in practice.

\section{References}

[1] M. Abudy and Y. Izhakian. Pricing stock options with stochastic interest rate. International Journal of Portfolio Analysis and Management, 1(3):250-277, 2013.

[2] C. M. Bender and S. A. Orszag. Advanced mathematical methods for scientists and engineers I. Springer Science \& Business Media, 1999.

[3] F. Black and M. Scholes. The pricing of options and corporate liabilities. The journal of political economy, pages 637-654, 1973. 
[4] D. Brigo and F. Mercurio. Interest rate models-theory and practice: with smile, inflation and credit. Springer Science \& Business Media, 2007.

[5] P. Carr and R. Lee. Realized volatility and variance: Options via swaps. Risk, 20(5):76-83, 2007.

[6] P. Carr and R. Lee. Robust replication of volatility derivatives. In PRMIA award for Best Paper in Derivatives, MFA 2008 Annual Meeting, 2008.

[7] R. J. Elliott and G.-H. Lian. Pricing variance and volatility swaps in a stochastic volatility model with regime switching: discrete observations case. Quantitative Finance, 13(5):687-698, 2013.

[8] M. Forde and A. Jacquier. Robust approximations for pricing Asian options and volatility swaps under stochastic volatility. Applied Mathematical Finance, 17(3):241$259,2010$.

[9] A. Grünbichler and F. A. Longstaff. Valuing futures and options on volatility. Journal of Banking $\mathscr{G}$ Finance, 20(6):985-1001, 1996.

[10] J. D. Hamilton. Analysis of time series subject to changes in regime. Journal of Econometrics, 45(1):39-70, 1990.

[11] X.-J. He and S.-P. Zhu. An analytical approximation formula for European option pricing under a new stochastic volatility model with regime-switching. Journal of Economic Dynamics and Control, 71:77-85, 2016.

[12] X.-J. He and S.-P. Zhu. How should a local regime-switching model be calibrated? Journal of Economic Dynamics and Control, 78:149-163, 2017.

[13] X.-J. He and S.-P. Zhu. A closed-form pricing formula for European options under the Heston model with stochastic interest rate. Journal of Computational and Applied Mathematics, 335:323-333, 2018. 
[14] X.-J. He and S.-P. Zhu. On full calibration of hybrid local volatility and regimeswitching models. Journal of Futures Markets, 38(5):586-606, 2018.

[15] S. Heston and S. Nandi. Derivatives on volatility: some simple solutions based on observables. 2000 .

[16] S. L. Heston. A closed-form solution for options with stochastic volatility with applications to bond and currency options. Review of Financial Studies, 6(2):327-343, 1993.

[17] S. Howison, A. Rafailidis, and H. Rasmussen. On the pricing and hedging of volatility derivatives. Applied Mathematical Finance, 11(4):317-346, 2004.

[18] J.-H. Kim, J.-H. Yoon, and S.-H. Yu. Multiscale stochastic volatility with the HullWhite rate of interest. Journal of Futures Markets, 34(9):819-837, 2014.

[19] T. Little and V. Pant. A finite difference method for the valuation of variance swaps. In Quantitative Analysis In Financial Markets: Collected Papers of the New York University Mathematical Finance Seminar (Volume III), pages 275-295. World Scientific, 2001.

[20] A. Van Haastrecht, R. Lord, A. Pelsser, and D. Schrager. Pricing long-dated insurance contracts with stochastic interest rates and stochastic volatility. Insurance: Mathematics and Economics, 45(3):436-448, 2009.

[21] S.-P. Zhu and G.-H. Lian. A closed-form exact solution for pricing variance swaps with stochastic volatility. Mathematical Finance, 21(2):233-256, 2011.

[22] S.-P. Zhu and G.-H. Lian. Analytically pricing volatility swaps under stochastic volatility. Journal of Computational and Applied Mathematics, 288:332-340, 2015. 